\title{
Experience of a novel community testing programme for COVID-19 in London: Lessons learnt
}

\author{
Authors: Gabriel Wallis, ${ }^{A}$ Francesca Siracusa, ${ }^{\mathrm{B}}$ Michael Blank, ${ }^{\mathrm{C}}$ Helena Painter, ${ }^{\mathrm{C}}$ Javier Sanchez, ${ }^{\mathrm{D}}$ Kelcy Salinas, ${ }^{\mathrm{D}}$ \\ Cherifer Mamuyac, ${ }^{D}$ Cindy Marudamuthu, ${ }^{\mathrm{E}}$ Fenella Wrigley, ${ }^{\mathrm{F}}$ Tumena Corrah,, ${ }^{\mathrm{G}}$ Tommy Rampling, ${ }^{\mathrm{H}}$ Sarah Logan, \\ Anna Goodman, ${ }^{3}$ Deborah Miller, ${ }^{\mathrm{K}}$ Bhanu Williams, ${ }^{\mathrm{L}}$ Alastair McGregor, ${ }^{\mathrm{G}}$ Victoria Parris, ${ }^{G}$ Gurjinder Sandhu, ${ }^{\mathrm{G}}$ \\ Laurence John, ${ }^{G}$ Padmasayee Papineni ${ }^{G}$ and Ashley Whittington ${ }^{G}$
}

We describe the London community testing programme developed for COVID-19, audit its effectiveness and report patient acceptability and patient adherence to isolation guidance, based upon a survey conducted with participants. Any patients meeting the Public Health England (PHE) case definition for COVID-19 who did not require hospital admission were eligible for community testing. 2,053 patients with suspected COVID-19 were tested in the community between January and March 2020. Of those tested, 75 (3.6\%) were positive. $88 \%$ of patients that completed a patient survey felt safe and $82 \%$ agreed that community testing was preferable to hospital admission. $97 \%$ were able to remain within their own home during the isolation period but just $41 \%$ were able to reliably isolate from other members of their household. The London community testing programme allowed widespread testing for COVID-19 while minimising patient transport, hospital admissions and staff exposures. Community testing was acceptable to patients and preferable to admission to hospital. Patients were able to reliably isolate in their home but not from household contacts. The authors believe in the importance, feasibility and acceptability of community testing for COVID-19 as a part of a package of interventions to mitigate a second wave of infection.

Authors: ${ }^{A}$ specialist registrar in infectious diseases, London North West University Healthcare NHS Trust, Harrow, UK; ${ }^{B}$ medical student, Imperial College London Faculty of Medicine, London, UK; Cjunior doctor in infectious diseases, London North West University Healthcare NHS Trust, Harrow, UK; Dsenior nurse in infectious diseases, London North West University Healthcare NHS Trust, Harrow, UK; Ematron in infectious diseases, London North West University Healthcare NHS Trust, Harrow, UK; F ${ }^{F}$ chief medical officer, London Ambulance Service NHS Trust, London, UK; ${ }^{6}$ consultant in infectious diseases, London North West University Healthcare NHS Trust, Harrow, UK; ${ }^{H}$ specialist registrar in infectious diseases, The Hospital for Tropical Diseases, London, UK; and University College London Hospitals NHS Foundation

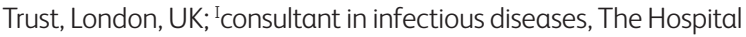
for Tropical Diseases, London, UK; and University College London

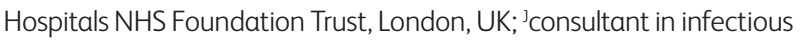
diseases, Guy's and St Thomas' NHS Foundation Trust, London, UK; Khead of nursing in integrated medicine, Guy's and St Thomas' NHS Foundation Trust, London, UK; ' 'consultant in paediatrics, London North West University Healthcare NHS Trust, Harrow, UK
KEYWORDS: COVID-19, community screening, self-isolation, HCID, survey

DOI: $10.7861 /$ clinmed.2020-0436

\section{Introduction}

Coronavirus disease 2019 (COVID-19) is an infectious disease caused by severe acute respiratory syndrome coronavirus 2 (SARS-CoV-2). ${ }^{1}$ First identified in Wuhan City, China, COVID-19 can range from a mild infection to severe disease with manifestations including pneumonia, acute respiratory distress syndrome and death. ${ }^{2}$ Since it was first identified in December 2019, COVID-19 has spread globally and is now recognised as a pandemic by the World Health Organization. ${ }^{3}$

In January 2020, Public Health England classified COVID-19 as a high consequence infectious disease (HCID). ${ }^{4}$ UK public health policy until the 13 March 2020 was one of 'containment', which necessitated rigorous testing and isolation of all suspected cases. Any patient with a cough, fever or shortness of breath developing within 14 days of travel to Wuhan City, China were referred to secondary care for assessment and testing. In the weeks that followed the case definition expanded to include mainland China (31 January), Hong Kong, Japan, Macau, Malaysia, Singapore, Republic of Korea, Taiwan and Thailand (7 February), and Italy, Iran, Vietnam, Myanmar and Laos (25 February). ${ }^{5}$

Initially, in London, patients with suspected COVID-19 could only be tested in secondary care, where the necessary clinical and infection prevention and control expertise was located. Safely transporting suspected cases to hospital for testing, and isolating them until results became available, was resource-intensive. Our initial experience was that the majority of patients with suspected COVID-19 had mild symptoms and did not require emergency ambulance or hospital admission. In order to overcome the inefficiency of the existing transfer-test-and-admit model, four NHS trusts - London North West University Healthcare NHS Trust (LNWUHT), University College London Hospitals NHS Trust, Guy's and St Thomas' NHS Foundation Trust and St George's University Hospitals NHS Foundation Trust - introduced a Londonwide community testing programme (see Fig 1). This process was supported and facilitated by the NHSE London Emergency Preparedness, Resilience and Response team. To the authors' 


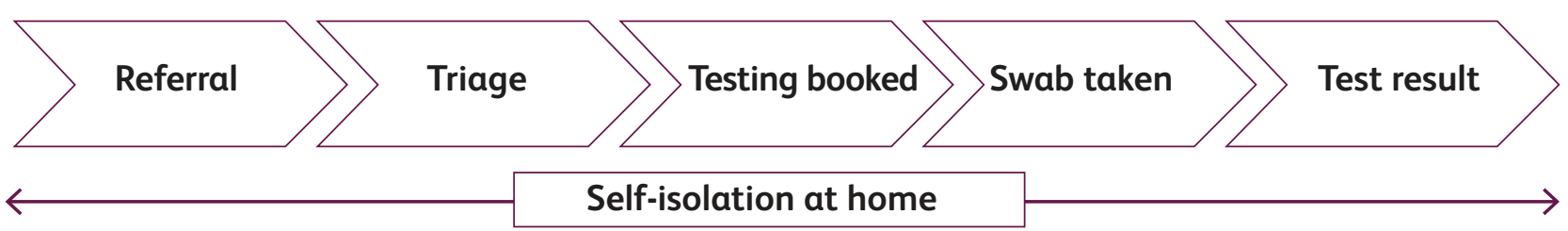

Fig 1. Community testing process.

knowledge, this was the UK's first community testing programme for COVID-19. Community testing has been used elsewhere during the COVID-19 pandemic, ${ }^{6}$ and was subsequently replicated in other areas of the UK where it was found to be pragmatic, safe and cost effective, ${ }^{7,8}$ however, there is an absence of literature on its acceptability to patients and patient-reported adherence to isolation guidelines.

We describe below the community testing protocol that we developed, audit its effectiveness, describe results of a patient satisfaction survey and highlight lessons learned which are relevant to the UK's public health strategy going forward in this, and in future pandemics.

\section{Methods}

\section{Testing protocol}

Patients with suspected COVID-19 were referred to community testing 'hubs' via the UK NHS 111 telephone service, London Ambulance Service (LAS) or other healthcare professionals. These hubs were based in secondary care. Each NHS trust had an individualised protocol (see Fig 1) but broadly patients were triaged by an infectious diseases (ID) doctor or nurse. Adult nurses were granted rapid extension of their scope of practice to triage children aged $>5$ years with the aid of a structured proforma developed by the paediatric team. Triage ensured that patients met the case definition, did not require in-hospital assessment, and were able to safely self-isolate at home. Video triage was also used in children aged $<5$ years. Testing was completed within $24-48$ hours of referral, either in the patient's home or via drive-through testing. Home testing was carried out in collaboration with LAS. Testing clinicians were transported to a patient's home by LAS before donning personal protective equipment (PPE) and taking a combined nose and throat swab. The testing clinician then exited the property, doffed PPE and returned to the community testing hub where potentially hazardous waste (used PPE) was disposed of. Drivethrough testing was carried out in a designated area of the hospital site; testing clinicians donned PPE and took a combined nose and throat swab, with the patient remaining in their car. All patients were given written information regarding self-isolation and an emergency contact number to call in the event of deterioration. Patients testing negative received a telephone call or text message communicating their results and advice on discontinuing isolation. Patients testing positive were discussed with Public Health England and managed according to their recommendations.

\section{Data analysis}

We performed a retrospective analysis of all cases referred to and tested by the community COVID-19 testing services operated by our four hospitals between 25 January 2020 and 13 March 2020.
In addition, we interrogated the LNWUHT community testing database to obtain demographic data, travel history, history of contact with confirmed cases, symptoms, medical history and test results for all patients tested at LNWUHT. For our analysis, where multiple countries were visited, the primary travel destination has been recorded for each patient.

\section{Patient survey}

As part of a quality improvement initiative, an anonymous patient survey was sent to patients aged over 18 years who were tested by LNWUHT. The survey investigated patient views on access, communication and acceptability of community testing as well as adherence to isolation guidelines. Patients were excluded if their COVID-19 result was pending or if the patient did not have a UK mobile phone number. Eligible patients were directed by text message to a survey hosted on an encrypted website (Qualtrics, Provo, Utah). Likert scales were used to assess patient satisfaction with communication, and acceptability of community testing. The full survey can be viewed in the supplementary material (S1). Descriptive analysis was performed on quantitative data.

\section{Ethics}

As this is a service evaluation project where no patient records were accessed for the purposes of research, formal NHS Research Ethics Approval was not deemed necessary. Local approval was obtained to ensure data management and the patient survey met NHS Information Governance requirements. Consent for the survey was implied by its completion.

\section{Results}

During the 'containment' phase, the rate of positive tests was low and the changing case definition led to surges in testing demand. Between 25 January 2020 and 13 March 2020, four NHS trusts (LNWUHT, University College London Hospitals NHS Trust, Guy's and St Thomas' NHS Foundation Trust and St George's University Hospitals NHS Foundation Trust) tested 2,053 community referrals for COVID-19, of which 1,116 (54\%) patients were tested in their own home. The remaining 937 patients were tested on the hospital sites, by drive-through testing. Of those tested, 75 (3.6\%) were positive. Demographic data and travel location were available for LNWUHT's 865 patients, which is shown in Table 1. Of patients tested at LNWUHT, 143 (16.5\%) were children aged 15 years and under. All of the patients screened for contact with a confirmed case were referred after 28 February, peaking on 10 March ( 44 people), reflecting the increasing global case number. Fig 2 shows the change in numbers of people tested as the course of the programme developed; changes in the case definition led to significant rises in the number of patients requiring testing. 
Table 1. Demographic data and screening reason for patients from London North West University Healthcare NHS Trust

\begin{tabular}{|c|c|}
\hline Characteristic & Number (\%) \\
\hline Total & $865(100)$ \\
\hline COVID-19-positive & $43(4.97)$ \\
\hline \multicolumn{2}{|l|}{ Gender } \\
\hline Male & $400(46.2)$ \\
\hline Female & $465(53.8)$ \\
\hline \multicolumn{2}{|l|}{ Age category } \\
\hline $0-15$ & $143(16.5)$ \\
\hline $16-30$ & $201(23.2)$ \\
\hline $31-45$ & $327(37.8)$ \\
\hline $46-60$ & $137(15.8)$ \\
\hline $61-75$ & $51(5.9)$ \\
\hline $75+$ & $6(0.69)$ \\
\hline \multicolumn{2}{|c|}{ Travel destination/reason screened } \\
\hline Italy & $273(31.5)$ \\
\hline Contact with a confirmed case & $198(22.9)$ \\
\hline Thailand & $110(12.7)$ \\
\hline Singapore & $48(5.5)$ \\
\hline Iran & $40(4.6)$ \\
\hline Information not available & $38(4.4)$ \\
\hline Malaysia & $37(4.2)$ \\
\hline China & $35(4.0)$ \\
\hline Japan & $31(3.6)$ \\
\hline Hong Kong & $29(3.4)$ \\
\hline Other & $12(1.4)$ \\
\hline South Korea & $10(1.2)$ \\
\hline Vietnam & $4(0.5)$ \\
\hline
\end{tabular}

The patient satisfaction survey was distributed to the first 333 participants of the LNWUHT community testing programme, of which 96 responses were received (29\%). A total of 62 of 95 respondents $(65 \%)$ reported that they were contacted by the local ID team on the same day as reporting their symptoms to a healthcare provider; 14 (15\%) waited two or more days to be contacted. A total of 48 out of 95 respondents (51\%) reported being tested on the day they were contacted by the ID team. Despite this rapid triage and testing, the majority $(n=89 ; 94 \%)$ waited two or more days for test results due to the turnaround time of results early in the pandemic.

Community testing helped inform patients, and $84 \%$ of participants $(n=76)$ strongly or somewhat agreed with the statement that they felt satisfied with the information they received and $87 \%$ of participants $(n=79)$ felt that they were given clear information about who to contact if their symptoms deteriorated. The majority $(n=80 ; 88 \%)$ of respondents reported feeling safe during the community testing programme. Finally, $70 \%$ of respondents $(n=63)$ strongly agreed that community or drive-through testing was preferable to hospital admission and isolation, and a further $12 \%(n=11)$ somewhat agreed. Nine of $90(10 \%)$ of respondents disagreed suggesting they would have preferred hospital admission.

Patients referred for community testing were able to isolate in their own home but not from other members of their household.

Survey respondents were asked to self-report the extent to which they adhered to the information they received on self-isolation. 89 of 92 respondents $(97 \%)$ were able to remain within their house during the isolation period. However, despite receiving written guidance to self-isolate in their own bedroom, less than half of the participants ( $n=38 ; 41 \%)$ were able to completely avoid contact with other members of their household.

\section{Discussion}

The London community testing programme was the first community testing programme for an HCID in the UK. This novel programme was designed to permit testing in an evolving pandemic in which transport and admission of patients was minimised, while patient and staff safety were maintained. We show that community testing was acceptable to patients and preferable to hospital admission. The survey also demonstrates that patients were reliably able to isolate in their own home, but not from other members of their household.

The London community testing programme tested 2,053 patients and identified 75 patients with COVID-19 facilitating case isolation and contact tracing during the 'containment' phase. Community testing was critical to relieving pressure on secondary care and ambulance services across London. The programme was rapidly developed, and was running effectively by 25 January 2020, 6 days prior to the first reported case in the UK. The majority of patients reported that community testing was a safe and acceptable method of testing for COVID-19. A minority of patients would have preferred to have been admitted to hospital for testing and isolation. This likely reflects an understandable concern for their own and their families' health.

This study has a number of potential limitations. Firstly, this was a retrospective study with no control group, and we are therefore unable to draw conclusions as to whether community testing is as effective as in-hospital testing in preventing transmission of COVID-19. Secondly, the patient survey was not piloted, only distributed to patients tested by LNWUHT, and had a response rate of $29 \%$. This may have meant that results are not representative or generalisable to other areas of the UK. Finally, the patient survey relied on self-reporting and was open to selfreporting bias.

There were several challenges to delivering community testing. Firstly, the number of patients requiring testing for COVID-19 was directly linked to the changing case definition (Fig 2). Each change led to surges in referrals which required rapid increases in community testing capacity. In future outbreaks, coordinating national case definition changes with testing services would allow preparation for a surge in referrals.

Secondly, delivering a community testing programme while continuing to provide inpatient and outpatient hospital services placed a huge burden on a small number of specialist ID doctors and nurses. These staff were working far beyond normal capacity at a time when their expertise was also required to develop local COVID-19 patient pathways, assist with structural reorganisation 


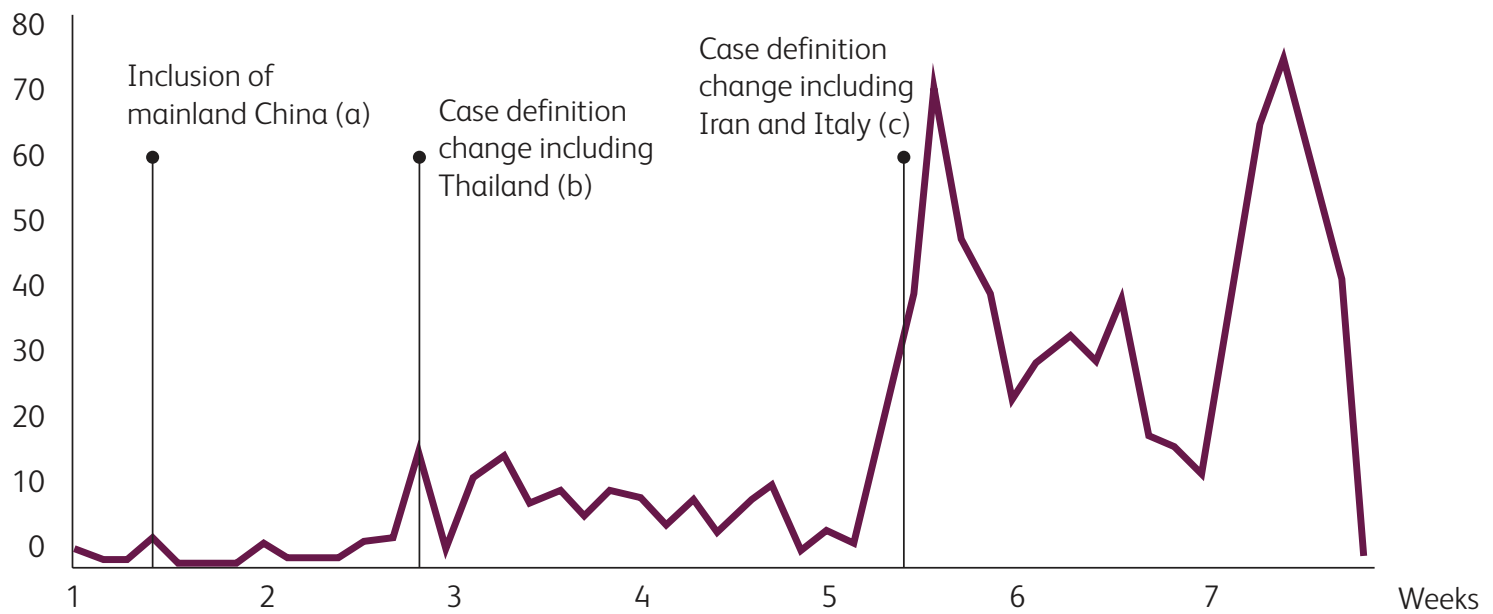

Fig 2. Graph showing number of people referred for testing by date, with labels indicating additions to the COVID-19 case definition by Public Health England, from commencement of screening on 25 January 2020 until the end of the programme on 13 March 2020. Key to full case definition changes: (a) Mainland China; (b) Hong Kong, Japan, Macau, Malaysia, Singapore, Republic of Korea, Taiwan, Thailand; (c) Italy, Iran, Vietnam, Myanmar and Laos.

of their hospitals, and train staff for PPE usage in preparation for the pandemic. In future outbreaks, if the community testing model is used, careful consideration must be given as to whether community testing is best delivered by already stretched secondary care services, or an alternative provider.

Finally, although patients tested in the community were able to isolate in their own home, they were unable to reliably isolate from other members of their household. Public health guidance at the time allowed household contacts to continue going to work, school and socialise with others, on the assumption they were not in direct contact with a patient with suspected COVID-19. Public health advice in the UK has now changed to mandate that all members of the household of a symptomatic case should self-isolate together. Our experience suggests this advice should have been in place from the start of the COVID-19 outbreak. Adherence to isolation guidance is easier for those privileged with access to their own bedroom, ability to work from home, and eligibility for sick pay.

At the time of writing, new cases, deaths, and hospital admissions due to COVID-19 are falling in the UK, and the easing of control measures are under way. Widespread community testing and isolation of cases and contacts is a key component of the government strategy to ease control measures while mitigating a second wave of infection (NHS Test and Trace). ${ }^{9}$ The authors believe in the importance, feasibility and acceptability of community testing for COVID-19 as part of a package of interventions to mitigate a second wave of infection. Community testing could also allow for clinical trials of antiviral medication in the early stages of infection, where they may be more effective, as with other viral infections such as influenza. ${ }^{10}$ The NHS Test and Trace service will need to be fully working, capable of dealing with surges in referrals, and locally responsive, for it to be effective. Household isolation-integral to a test and isolate approach — will have an impact on the ability of many to work or care for dependents. This could present significant financial and social challenges for many and may disproportionately affect those with little social support and lower-income households, representing a major barrier to adherence.
In summary, we describe the experience of the London community testing programme in the evolving COVID-19 pandemic and the systems that we designed to allow us to test at volume, while minimising patient transport, hospital admissions and staff exposures. Community testing was found to be more acceptable to patients than alternatives.

\section{Supplementary material}

Additional supplementary material may be found in the online version of this article at www.rcpjournals.org/clinmedicine: S1 - COVID-19 Community Testing Questionnaire.

\section{Acknowledgements}

We would like to acknowledge the infectious diseases department of St George's University Hospital NHS Foundation Trust who provided community testing data for this paper. We would like to acknowledge the hard work of all the doctors, nurses and paramedics who helped to deliver community testing. Without their hard work none of this would have been possible.

\section{References}

1 World Health Organization. Naming the coronavirus disease (COVID-19) and the virus that causes it. WHO, 2020. Available from www.who.int/emergencies/diseases/novel-coronavirus-2019/ technical-guidance/naming-the-coronavirus-disease-(covid-2019)and-the-virus-that-causes-it [Accessed 1 June 2020].

2 Zhang J, Dong X, Cao Y et al. Clinical characteristics of 140 patients infected with SARS-CoV-2 in Wuhan, China. Allergy 2020, in press (doi: 10.1111/all.14238).

3 World Health Organization. WHO director-general's opening remarks at the media briefing on COVID-19 - 11 March 2020. www.who.int/ dg/speeches/detail/who-director-general-s-opening-remarks-at-themedia-briefing-on-covid-19-11-march-2020 [Accessed 1 June 2020].

4 Public Health England. COVID-19: guidance for health professionals. www.gov.uk/government/collections/wuhan-novel-coronavirus [Accessed 25 January 2020]. 
5 Public Health England. Investigation and initial clinical management of possible cases of novel coronavirus (2019-Ncov) infection. PHE, 2020. www.gov.uk/government/publications/wuhan-novel-coronavirusinitial-investigation-of-possible-cases/investigation-and-initial-clinicalmanagement-of-possible-cases-of-wuhan-novel-coronavirus-wncov-infection [Accessed 6 February 2020]

6 Kwon K, Ko J, Shin $\mathrm{H}$ et al. Drive-through screening center for COVID-19: a safe and efficient screening system against massive community outbreak. J Korean Med Sci 2020;35:e123.

7 Mark K, Steel K, Stevenson J et al. Coronavirus disease (COVID-19) community testing team in Scotland: a 14-day review, 6 to 20 February 2020. Euro Surveill 2020;25:2000217.

8 Hill K, Campbell R, Mutch C et al. Drive-through testing in COVID-19: experience from NHS Lothian. Clin Med 2020;20:290-1.
9 Department of Health and Social Care. NHS test and trace: how it works. www.gov.uk/guidance/nhs-test-and-trace-how-it-works [Accessed 1 June 2020].

10 Aoki F, Macleod M, Paggiaro P et al. 2002. Early administration of oral oseltamivir increases the benefits of influenza treatment. J Antimicrob Chemother 51:123-9.

Address for correspondence: Dr Ashley Whittington, Department of Infectious Diseases, London North West University Healthcare NHS Trust, Northwick Park Hospital, Watford Road, Harrow, Middlesex HA1 3UJ, UK.

Email: a.whittington@nhs.net 